\title{
COMPOSIÇÃO QUÍMICA, ATIVIDADE ANTIRADICALAR E ANTIMICROBIANA DO PÓLEN APÍCOLA DE FABACEAE
}

Francisco R. L. Caldas ${ }^{\mathrm{a}}$, Francisco Augusto Filho ${ }^{\mathrm{b}}$, Heberty T. Facundo ${ }^{\mathrm{c}}$, Rodolfo F. Alves ${ }^{\mathrm{d}}$, Francisco de A. R. dos Santos ${ }^{\mathrm{d}}$, Girliane R. da Silva ${ }^{e}$, Celso A. Camara ${ }^{e}$ e Tania M. S. Silva ${ }^{\mathrm{e}, *,()}$

anstituto Federal de Educação, Ciência e Tecnologia, 63040-540 Juazeiro do Norte - CE, Brasil

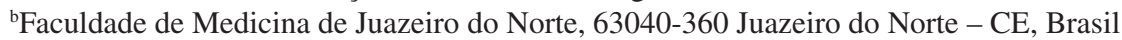

'Universidade Federal do Cariri, 63180-000 Barbalha - CE, Brasil

${ }^{d}$ Departamento de Ciências Biológicas, Universidade Estadual de Feira de Santana, 44036-900 Feira de Santana - BA, Brasil

'Departamento de Química, Universidade Federal Rural de Pernambuco, 52171-900 Recife - PE, Brasil

Recebido em 03/06/2018; aceito em 25/10/2018; publicado na web em 07/11/2018

\begin{abstract}
CHEMICAL COMPOSITION, ANTIRADICALAR AND ANTIMICROBIAL ACTIVITY OF FABACEAE POLLEN BEE. The aim of this investigation was to analyze two samples of pollen collected by Apis mellifera bees in view of their chemical composition, antiradicalar and antimicrobial activities. Palynological analysis showed that the predominant pollen was Fabaceae species (42.3\%, sample I and $50.6 \%$, sample II). The characterization by ultra-performance liquid chromatography coupled with a diode array detector and quadrupole time of flight mass spectrometry (UPLC-DAD-QTOF-MS/MS) resulted in the identification of three derivatives of putrescine (di-4-coumaroylputrescine, coumaroyl-feruloyl-putrescine and di-feruloyl-putrescine), two spermidine derivatives (coumaroyl-di-cafeoyl-spermidine and tri-coumaroyl-spermidine and four biflavonoids (2,3-di-hydrohydroxy-amentoflavone, 2,3-dihydro-amentoflavone, amentoflavone and 2,3-dihydro-biapigenin). The two samples showed antiradical and antimicrobial activities, probably due to the presence of these compounds.
\end{abstract}

Keywords: pollen bee; Apis mellifera; antioxidants; antimicrobials.

\section{INTRODUÇÃO}

O pólen apícola é um dos mais ricos e puros suplementos alimentares naturais embalados por abelhas em grânulos e, posteriormente, colhidos a partir de colmeias por seres humanos. ${ }^{1}$ É o resultado da aglutinação do pólen das flores, néctar e substâncias salivares das abelhas, ${ }^{2}$ coletado por meio de uma grade de retenção denominado coletor de pólen. No final da coleta encontram-se reunidas as bolotas de grãos de coloração variável, indicando as diversas espécies botânicas colecionadas pelas abelhas, formando uma mistura conhecida por "mix" polínico, sendo esse material removido pelo apicultor para o beneficiamento, comercialização e consumo animal e humano. ${ }^{3}$

A apicultura é uma prática de grande potencial no Brasil devido a fatores favoráveis como o clima e a diversidade da flora. ${ }^{4}$ É uma das poucas atividades agropecuárias que atende aos três requisitos da sustentabilidade: o econômico, o social e o ecológico. Sendo assim, fornece renda para o apicultor, ocupa mão de obra familiar ou contratada e contribui para a preservação da flora nativa, pois é dela que são extraídos o néctar e o pólen, componentes essenciais para a vida das colméias. ${ }^{4}$ No nordeste brasileiro ainda predomina a obtenção de pólen apícola obtido da flora nativa. ${ }^{5,6}$ É uma das poucas regiões no mundo com o potencial para produzir produtos apícolas orgânicos em grande quantidade, devido à diversidade de espécies de plantas e microclimas, juntamente com vastas áreas de terras inexploradas, livre da atividade agrícola convencional. ${ }^{7}$

Em geral, os apicultores coletam o pólen misto (heterofloral ou multifloral), em virtude da diversidade da flora, no entanto, em determinadas épocas do ano pode ocorrer a predominância de uma espécie em floração em detrimento de outras, possibilitando a coleta do pólen unifloral ou monofloral, que apresentará uma alta frequência de pólens de uma mesma espécie botânica. Sendo assim,

*e-mail: sarmentosilva@gmail.com o pólen monofloral, que apresenta predominância do pólen de uma mesma espécie vegetal (maior que 45\%), mantém as propriedades organolépticas e bioquímicas semelhantes às da planta inicial, ao passo que o pólen multifloral tem propriedades variáveis de mais do que duas plantas originais. ${ }^{8,9}$ Do ponto de vista da padronização, o pólen monofloral é importante porque somente este tipo de pólen tem composição constante e, portanto, pode ser usado com sucesso na nutrição e na medicina. ${ }^{1,9}$

Os componentes majoritários do pólen apícola são açúcares (13$55 \%)$, fibras (0,3-20\%), proteínas (10-40\%) e lipídeos (1-10\%). ${ }^{10,11}$ Outros componentes em menor quantidade são minerais (cálcio, manganês, magnésio, fósforo, selênio, zinco, cobre, ferro, potássio e sódio), ${ }^{7}$ vitaminas (tiamina, riboflavina, niacina, ácido pantotênico, piridoxina, ácido ascórbico, biotina, ácido fólico e tocoferol), ${ }^{12}$ carotenoides, compostos fenólicos, em especial os flavonoides, ${ }^{6,11,13}$ esteroides e terpenos. ${ }^{1}$

A composição química do pólen pode variar dependendo da origem vegetal e geográfica e o conjunto de outros fatores como condições climáticas, tipo de solo e das atividades do apicultor. ${ }^{1,10}$ Cada tipo de pólen tem suas próprias características específicas relacionadas com a genética das espécies de flores e plantações visitadas pelas abelhas e que podem influenciar nas propriedades biológicas. ${ }^{11,14}$ É provável que a composição do pólen apícola não se limite as proporções de diferentes flores no meio, mas sim, pelo menos até certo ponto, seja determinada pela preferência das abelhas. ${ }^{1,15}$

O consumo de pólen apícola ou de seus produtos derivados, como um suplemento dietético por seres humanos, tem crescido rapidamente. Além disso, o interesse sobre o pólen vem aumentando, devido ao seu conteúdo nutricional e às atividades biológicas. ${ }^{1,11,16}$

Além dos polens apícolas apresentarem potencial utilização na área farmacêutica, podem também ser agentes protetores eficientes para uso como antioxidantes naturais e antibacterianos em sistemas alimentícios, sendo estas atividades dependentes da composição química, que, por 
sua vez, depende da diversidade geográfica e da origem botânica. Geralmente, as atividades antioxidantes e antimicrobianas do pólen apícola são atribuídas aos componentes fenólicos presentes. ${ }^{12,17-19}$

Este trabalho é continuação dos estudos com os produtos apícolas e meliponícolas, ${ }^{6,20-27}$ sendo apresentadas as análises físico-químicas, químicas por Cromatografia a Líquido de Ultra-eficiência acoplada com detectores de Arranjo de Diodo e Espectrômetro de Massas tipo triploquadrupolo e Tempo de Vôo (UPLC-DAD-qTOF-MS/MS), atividades antirradicalar e antimicrobiana da fração fenólica do pólen apícola de Fabaceae.

\section{PARTE EXPERIMENTAL}

\section{Equipamentos e reagentes}

Para os testes físico-químicos foram utilizados balança analítica (AW220, Shimadzu, Kyoto, Japan), balança de infravermelho (ID50, Marte, Pernambuco, Brasil), pHmetro (TEC-3MP, Tecnal, São Paulo, Brasil), mufla (1P 7000, Edgcon, São Paulo, Brasil), bloco digestor (TE-040/25GE, Tecnal, São Paulo, Brasil) e equipamento medidor da atividade de água (AquaLab 4TE, Decagon Devices, São Paulo, Brasil.

Para as análises de Cromatografia Líquida de Alta Eficiencia (HPLC), utilizou-se o cromatógrafo (Shimadzu Prominence LC20AT, Shimadzu, Kyoto, Japan), constituído por duas bombas LC6AT, degaseificador DGU-20As, forno de coluna CTO-20AC, detector Indice de Refração RID-10A, injetor manual Rheodyne 7125i, com um loop $20 \mu \mathrm{L}$, ou autoinjetor (SIL-20A) e módulo de comunicação CBM-20A, controlado pelo software LcSolution. Foram usados filtros de membrana com poros de $0,45 \mu \mathrm{m}$ de diâmetro (Supelco) para filtração das amostras e dos solventes: metanol, (J. T. Baker, Phillipsburger, EUA) e água Mili-Q (Millipore, EUA). A análise espectrofotométrica para determinação das atividades antirradicalares foi realizada no aparelho (Asys HiTech UVM 340, Biochrom, EUA), utilizando placa de 96 poços. Foram utilizados os seguintes reagentes: Folin-Ciocalteu, DPPH (2,2-difenil-1-picril-hidrazila), ABTS [2,2'-azinobis- (ácido 3-etilbenzotiazolina-6-sulfônico) sal de diamônio 98\%], ácido gálico, ácido ascórbico (Sigma-Aldrich, Sternheim, Alemanha), carbonato de sódio e persulfato de potássio (Vetec, Rio de Janeiro, Brasil). As análises por UPLC-DAD-qTOF-MS/MS foram realizadas utilizando espectrômetro de massas XEVO-G2XSQTOF (Waters, Manchester, UK) conectado ao sistema ACQUITY UPLC (Waters, Milford, MA, USA) via ionização por eletrospray (ESI) e detector analítico DAD (Waters Acquity), comprimento de onda 200-400 nm.

\section{Coleta das amostras, determinação da origem botânica (análise} palinológica) e extração

Duas amostras de pólen apícola desidratado de colméias de abelhas Apis mellifera foram coletadas no estado da Bahia, Nordeste do Brasil. As amostras foram moídas e armazenadas a $-4^{\circ} \mathrm{C}$. As análises palinológicas foram realizadas no Laboratório de Micromorfologia Vegetal, Departamento de Ciências Biológicas da Universidade Estadual de Feira de Santana. As análises seguiram a aplicação da acetólise de Erdtman (1960) ${ }^{28}$ Os tipos polínicos foram identificados por comparação com lâminas depositadas na palinoteca do Laboratório de Micromorfologia Vegetal e com o auxílio dos catálogos polínicos disponíveis. Os tipos polínicos foram classificados como pólen dominante $(>45 \%)$, pólen acessório $(\leq 45 \% \geq 15 \%)$, pólen isolado importante $(\leq 15 \% \geq 3 \%)$ e pólen isolado ocasional $(<3 \%)$.

Para a extração dos constituintes fenólicos, as amostras I (145,1 g) e II (180,6 g) foram extraídas com etanol. As soluções extrativas foram evaporadas em rotaevaporador fornecendo o extrato etanólico (amostra $\mathrm{I}=80,7 \mathrm{~g}$ e amostra II=57,9 g). Para extração dos fenólicos,
10,0 g de cada amostra foram solubilizadas com metanol:água (1:1) e extraídas com hexano e acetato de etila. Os solventes foram evaporados e forneceram as frações hexânica (amostra $\mathrm{I}=0,9 \mathrm{~g}$ e amostra $\mathrm{II}=0,8 \mathrm{~g}$ ), acetato de etila rica em fenólicos ( $\operatorname{amos} t r a \mathrm{I}=3,3 \mathrm{~g}$ e amostra $\mathrm{II}=1,5 \mathrm{~g}$ ) e metanólica aquosa (amostra $\mathrm{I}=5,5 \mathrm{~g}$ e amostra $\mathrm{II}=6,5 \mathrm{~g})$.

\section{Testes físico-químicos}

A análise das características sensoriais foi realizada pelos métodos visual, olfativo e gustativo. Os testes foram realizados de acordo com o regulamento técnico para fixação de identidade e qualidade de pólen apícola. ${ }^{2} \mathrm{~A}$ determinação da umidade foi feita pelo método da perda por dessecação, utilizando uma balança de infravermelho, à $105^{\circ} \mathrm{C}$ por 30 minutos. A determinação potenciométrica do $\mathrm{pH}$ e da acidez livre foi realizada em $\mathrm{pHmetro.} \mathrm{A} \mathrm{medida} \mathrm{da} \mathrm{acidez} \mathrm{livre} \mathrm{foi}$ obtida pela titulação da suspensão com hidróxido de sódio $0,05 \mathrm{~N}$ até o ponto de equivalência ( $\mathrm{pH} 8,5)$. O teor de cinzas foi determinado pelo método de resíduo por incineração, para o qual pesaram-se 2,0 $\mathrm{g}$ da amostra em uma cápsula de porcelana, que foram previamente carbonizados em chapa aquecedora e, em seguida, incinerados em mufla à $550^{\circ} \mathrm{C}$, por 4 horas ou até eliminação completa do carvão. A atividade de água (Aw) foi feita utilizando um equipamento medidor da atividade de água (AquaLab 4TE).

\section{Determinação de açúcares por HPLC-RI}

Para análises dos açucares presentes no pólen apícola, as amostras foram extraídas com água e os extratos aquosos foram liofilizados. As soluções dos extratos aquosos foram preparadas na concentração de 40,0 $\mathrm{mg} \mathrm{mL}^{-1}$, diluídas com a fase móvel e filtradas. Utilizou-se uma coluna (Rezex RCM - Monosacarídeo $\mathrm{Ca}^{2+}(8 \%), 300 \mathrm{~mm} \times$ 7,8 mm x $8 \mu \mathrm{m}$, Phenomenex), temperatura de $85^{\circ} \mathrm{C}$, fluxo de $0,6 \mathrm{~mL} \mathrm{~min}{ }^{-1}$ e como fase móvel água com $5 \%$ de $\mathrm{MeOH}$, em modo isocrático. Para quantificação, foi feita a curva de calibração dos padrões glicose e frutose nas concentrações 20,$0 ; 10,0 ; 5,0 ; 2,5$ e $1,25 \mathrm{mg} \mathrm{mL}^{-1}$. O volume injetado foi de $20 \mu \mathrm{L}$ para padrão e amostras. A detecção foi feita com um detector de índice de refração.

\section{Determinação do teor de fenólicos totais e atividade antiradicalar}

O teor de fenólicos totais foi realizado de acordo com o método de Folin-Ciocalteau. ${ }^{29}$ Brevemente, $50 \mu \mathrm{L}$ da fração AcOEt dos polens apícolas foram misturados com $500 \mu \mathrm{L}$ do reagente de FolinCiocalteau e $500 \mu \mathrm{L}$ de $\mathrm{Na}_{2} \mathrm{CO}_{3}\left(1,0 \mathrm{mg} \mathrm{mL}^{-1}\right)$ e deixados no escuro por uma hora. Após este tempo a absorbância foi lida em $760 \mathrm{~nm}$. Foi construída uma curva com ácido gálico.

O teste com o DPPH foi realizado de acordo com a metodologia descrita por Silva et al. ${ }^{20}$ As soluções das frações AcOEt foram preparadas na concentração de $5,0 \mathrm{~g} \mathrm{~mL}^{-1}$. Foram adicionadas concentrações apropriadas da solução de DPPH $\left(23,6 \mu \mathrm{g} \mathrm{mL} \mathrm{m}^{-1} \mathrm{em} \mathrm{EtOH}\right)$ o volume foi completado com EtOH. Como controle positivo foi utilizado o ácido ascórbico. Após 30 minutos de agitação em aparelho de ultrassom ao abrigo da luz, a quantidade de radicais de DPPH foi registrada em espectrofotômetro UV-Vis no comprimento de onda de $517 \mathrm{~nm}$. A percentagem da atividade sequestradora foi calculada pela equação: \% $\mathrm{AS}=100 \times \Delta \mathrm{hx} / \mathrm{hc}, \mathrm{hc}=$ absorbância controle, $\mathrm{hx}=$ absorbância teste, $\Delta \mathrm{hx}=\mathrm{hc}-\mathrm{hx}$. Foi utilizado ácido ascórbico como controle positivo.

O ensaio de descoloração do ABTS foi realizado de acordo com Silva et al. ${ }^{20} \mathrm{O}$ radical ABTS foi preparado adicionando $5 \mathrm{~mL}$ da solução de ABTS 7 mmol L-1 com $88 \mu \mathrm{L}$ da solução de perssulfato de potássio $140 \mathrm{mmol} \mathrm{L}^{-1}$, reagindo durante 14 horas ao abrigo da 
luz. Para o ensaio, $1 \mathrm{~mL}$ da solução do radical ABTS foi diluída em EtOH até obter uma absorbância $\pm 0,7$ a $734 \mathrm{~nm}$. Foram preparadas soluções das amostras na concentração de 5,0 $\mathrm{g} \mathrm{mL}^{-1}$. Quantidades apropriadas foram misturadas com a solução de ABTS. Como controle positivo foi utilizado o trolox. Após 6 minutos de agitação em aparelho de ultrassom ao abrigo da luz a quantidade de radicais de ABTS foi registrada em espectrofotômetro UV-Vis no comprimento de onda de $734 \mathrm{~nm}$. A percentagem da atividade sequestradora foi calculada pela equação: $\% \mathrm{AS}=100 \mathrm{x} \Delta \mathrm{hx} / \mathrm{hc}, \mathrm{hc}=$ absorbância controle, $\mathrm{hx}=$ absorbância teste, $\Delta \mathrm{hx}=\mathrm{hc}-\mathrm{hx}$.

\section{Análises por UPLC-DAD-qTOF-MS/MS}

As separações cromatográficas foram realizadas utilizando uma coluna Acquity BEH C18 (2,1 x 50 mm, 1,7 $\mu \mathrm{m}$, Waters, EUA) a 40 ${ }^{\circ} \mathrm{C}$. A fase móvel binária consistiu de água com $0,1 \%$ de ácido fórmico (fase móvel A) e acetonitrila com $0,1 \%$ de ácido fórmico (fase móvel B). O fluxo foi de $0,4 \mathrm{~mL} / \mathrm{min}$ e o volume de injeção foi de $5,0 \mu \mathrm{L}$. O gradiente de eluição utilizado foi: 0,0-8,0 min 10\%-50\% de B; 8,09,0 min, $50 \%-95 \%$ de B e em 9,1 min $10 \%$ de B, o monitoramento foi feito a $340 \mathrm{~nm}$. O espectrômetro de massas foi operado em modo negativo de ionização (ESI-) no modo sensibilidade. A detecção foi realizada no modo centróide $\mathrm{MS}^{\mathrm{E}}$ em uma faixa de massa escolhida entre os valores de $m / z$ 50-1200 Da. Todas as análises foram realizadas utilizando o lockspray para garantir a precisão e reprodutibilidade dos valores de massas. Leucina-encefalina $\left(10 \mathrm{ng} \mathrm{mL}^{-1}\right)$ foi utilizado como padrão/referência para calibração. A aquisição e análise dos dados foi realizada utilizando o software Waters MassLynx.

\section{Determinação da Concentração Inibitória Mínima (CIM)}

A avaliação da atividade antibacteriana foi realizada a partir do método de microdiluição em caldo, modificado a partir do documento da CLSI/NCCLS M7-A6 para bactérias. ${ }^{30}$ Foram utilizadas linhagens padrão (ATCC), sendo uma Gram-positiva: Staphylococcus aureus ATCC 6538 e Gram-negativa: Escherichia coli ATCC 25922. Para a avaliação da atividade antifúngica foram utilizadas linhagens padrão provenientes da American Type Culture Collection (ATCC), sendo avaliadas Candida albicans ATCC 36232, C. glabrata ATCC 90030, C. krusei ATCC 34135, C. tropicalis ATCC 13803. As leveduras foram cultivadas e mantidas em meio ágar Sabouraud. A atividade antifúngica dos extratos foi determinada através do método de microdiluição em caldo, conforme protocolo M27-A2 do Clinical and Laboratory Standards Institute (CLSI - National Comittee for Clinical Laboratory Standards - NCCLS). ${ }^{30}$ As amostras foram testadas nas concentrações de 1024, 512, 256, 128, 64, 32, 16, $8 \mu \mathrm{g} \mathrm{mL} \mathrm{mL}^{-1}$, onde volumes de $100 \mu \mathrm{L}$ da suspensão leveduriforme foram inoculados em microplacas contendo $100 \mu \mathrm{L}$ Sabouraud, incubadas a $37^{\circ} \mathrm{C}$ por $48 \mathrm{~h}$, seguindo o processo de diluição seriada, foi retirada $100 \mu \mathrm{L}$ da cavidade mais concentrada para a cavidade sucessora. Os ensaios foram realizados em triplicata para cada amostra.

\section{Análises estatísticas}

Todas as amostras foram analisadas em triplicata e os resultados expressos como média \pm desvio padrão da média. Foi utilizado o programa Microsoft Excel software package (Microsoft Corp., Redmond, WA).

\section{RESULTADOS E DISCUSSÃO}

Os resultados das análises palinológicas para as duas amostras mostraram a predominancia de pólens da família Fabaceae. A amostra
I apresentou percentual de $42,3 \%$ e a amostra II 50,6\%, sendo essa última amostra considerada monofloral (pólen apícola com mais de $45 \%$ de grãos de pólen de uma mesma espécie botânica). Nove famílias de espécies vegetais apareceram nas duas amostras de pólen (Anacardiaceae, Aquifoliaceae, Arecaceae, Asteraceae, Dilleniaceae, Euphorbiaceae, Fabaceae, Malpighiaceae e Sapindaceae). O segundo pólen mais predominante nas duas amostras foi da Familia Dilleniaceae (26,6 \% para I e 14,3\% para II). O gráfico com a percentagem dos polens está na Figura 1.

Os dados sobre a origem botânica para o pólen apícola são muito importantes porque permitem o entendimento sobre as interações ecológicas entre as plantas e os polinizadores. A identificação taxonômica sobre as espécies que compõem a flora de uma determinada região fornece informações essenciais sobre os recursos naturais disponíveis e as características ecológicas de um determinado habitat ou ecossistema, além disso, a identificação das plantas apícolas é de extrema importância para os apicultoes, pois indica as fontes alimentares utilizadas para a coleta de néctar e pólen pelas abelhas. ${ }^{31}$ É interessante notar que os produtos apícolas oriundos da caatinga apresentam em sua maioria a predominância de pólens de espécies de Fabaceae, pois espécies dessa família são comumente encontradas na região Nordeste, principalmente em regiões secas como a caatinga. ${ }^{32}$ Vinte e duas amostras da própolis produzida por Apis mellifera em uma região do semiárido no Estado da Bahia (Agreste de Alagoinhas), Brasil, foram analisadas palinologicamente e a família Fabaceae foi a mais representativa neste estudo com nove tipos de polens, sendo o tipo Mimosa pudica presentes em todas as amostras analisadas. ${ }^{32}$ Amostras de polens ${ }^{20,27}$ e méis ${ }^{22}$ coletados pelas abelhas sem ferrão jandaíra (Melipona subnitida) também apresentaram predominancia de polens de espécies de Fabaceae, com preferencia para espécies do gênero Mimosa (comumente conhecidas como "jurema").33

As análises físico-químicas dos polens apícolas são importantes para atestar os padrões de qualidade, principalmente para polens monoflorais. De acordo com a origem polínica, estação e a região de coleta, o pólen pode apresentar uma composição variável, como teor de lipídeos, proteínas, açucares, etc. Para a regulamentação dos testes físico-químicos, no Brasil existe o Anexo V da Instrução Normativa $\mathrm{n}^{\mathrm{o}} 3$, de 19 de janeiro de $2001,{ }^{2}$ que é um regulamento técnico para fixação de identidade e qualidade de pólen apícola. Para as duas amostras do pólen de Fabaceae foram realizados os testes físico-químicos e os resultados estão na Tabela 1 . Na análise sensorial as amostras apresentaram grãos heterogêneos, de forma e tamanhos variados, tendendo a esféricos, cor amarelo claro, aroma característico e sabor doce, livres de impurezas como partes de abelha, cera, partículas de plantas ou outras matérias estranhas. Os parâmetros avaliados atendem às especificações exigidas pelo Ministério da Agricultura, Pecuária e Abastecimento (MAPA), com exceção da umidade, cujo teor é resultante do processo de secagem empregado nos apiários e passível de ajustes para adequação. $\mathrm{O}$ atendimento à norma do MAPA é o primeiro passo para comercialização do pólen apícola no mercado.

A análise e quantificação dos açucares livres nas duas amostras do pólen apícola foi realizada por HPLC-IR. Os dois principais açucares do pólen de Fabaceae são a glicose e frutose e a quantidade em miligramas por grama de pólen de glicose foi de 76,9 $\pm 19,56 \mathrm{mg} \mathrm{g}^{-1}$, na amostra I e 104,6 $\pm 6,40 \mathrm{mg} \mathrm{g}^{-1}$ na amostra II. Já a quantidade frutose foi de 76,9 $\pm 19,79 \mathrm{mg} \mathrm{g}^{-1}$ na amostra I e 103,4 $\pm 1,97 \mathrm{mg} \mathrm{g}^{-1}$ na amostra II (Tabela 2).

Os açúcares correspondem em média a 35\% da composição do pólen apícola, sendo o maior contribuinte para o valor energético total. $\mathrm{O}$ néctar é a fonte principal de açúcares na dieta das abelhas, mas o pólen das flores também contém açúcares solúveis simples constituídos predominantemente por mono e dissacarídeos. ${ }^{34}$ Nossos 


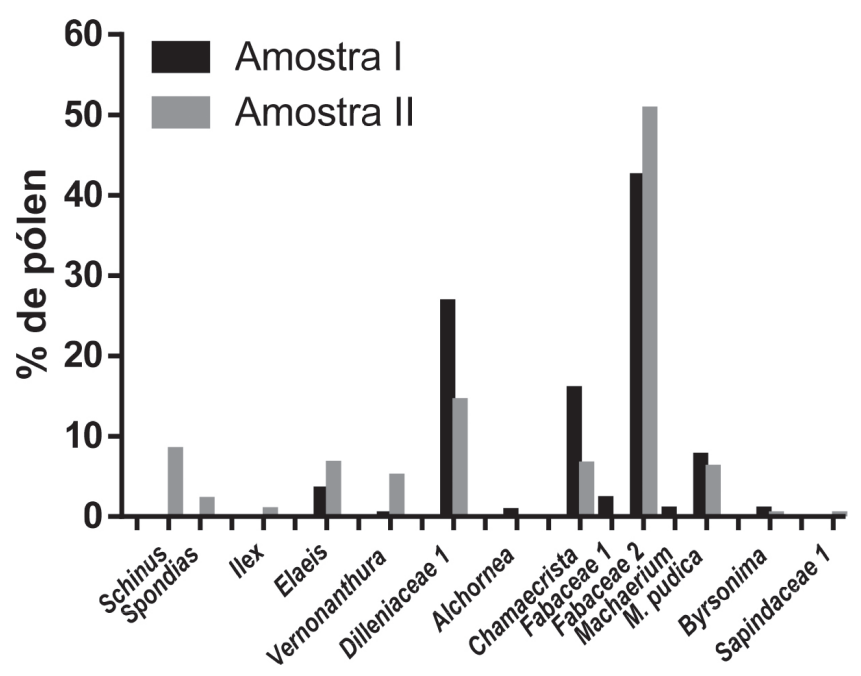

Figura 1. Espécies vegetais encontradas nas amostras de pólen através das análises palinológicas

resultados estão em conformidade com a literatura, cujos estudos demonstram que frutose e glicose são os açúcares livres presentes em maiores concentrações no pólen apícola (Apis mellifera), enquanto a sacarose pode estar presente em menores quantidades. Possivelmente, a hidrólise enzimática da sacarose realizada pelas abelhas contribui para o elevado teor de monossacarídeos. ${ }^{35} \mathrm{~A}$ composição dos carboidratos foi analisada em 28 amostras de pólens apícolas da Slovenia, destas, 10 amostras foram classificadas como monoflorais. Os monossacarídeos representaram $96 \%$ da fração de açucares, com uma variação de 13,2-27,8 $\mathrm{g}$ por $100 \mathrm{~g}$ do peso seco para frutose e $10,6-28,5 \mathrm{~g}$ por $100 \mathrm{~g}$ do peso seco para glicose. ${ }^{36}$

Os compostos fenólicos foram tentativamente identificados no pólen apícola de Fabaceae por UPLC-DAD-ESI-QTOF/MS/MS. As duas amostras apresentaram um perfil químico semelhante. Na Tabela 3 estão dispostos os dados obtidos pela análise dos espectros dos íons produtos de cada substância. Os espectros de massas das substâncias estão nas Figuras $1 \mathrm{~S}$ a 10S. Foram identificados 9 compostos fenólicos, sendo três derivados da putrescina $(\mathbf{1}, \mathbf{2}$ e $\mathbf{3})$, dois derivados da espermidina ( $\mathbf{4}$ e $\mathbf{6})$ e quatro biflavonoides $(\mathbf{5}, \mathbf{7}, \mathbf{8}$ e 9). Os compostos $1,2,3,4$ e 6 mostraram absorções típicas no UV indicativos de derivados de ácidos cinâmicos (Tabela 3). O composto 1 (tR 3,90 min, $\lambda \max =293 \mathrm{~nm}$ ) apresentou o pico da molécula desprotonada em $\mathrm{m} / \mathrm{z}$ 379,1661 [M-H] $]^{-}$em ESI no modo negativo com o íon produto em $\mathrm{m} / \mathrm{z}, 162,0560$ [M-H-coumaroil- $\left.\mathrm{C}_{4} \mathrm{H}_{7} \mathrm{~N}\right]^{-}$característico de um derivado de ácido cinâmico. Baseado nestes dados e com os valores dos íons produtos em 259,1336 [M-H-(4-etenil-fenol)]', 233,1336
[M-H-coumaroil] foi possível identificar 1 como sendo um conjugado de ácido cinâmico com a putrescina, di-4-coumaroilputrescina. ${ }^{37} \mathrm{Os}$ compostos $2(\mathrm{tR} 4,10 \mathrm{~min}, \lambda \max =308 \mathrm{~nm})$ e $3(\mathrm{tR} 4,27 \mathrm{~min}, \lambda \max =$ $317 \mathrm{~nm}$ ) apresentaram os picos das moléculas desprotonadas em $\mathrm{m} / z$ 409,1764 [M-H] - e m/z, 439,1874 [M-H] $]^{-}$. Quando comparados com a substancia 1 (di-4-coumaroil-putrescina), apresentam diferença de $\mathrm{m} / \mathrm{z}$ $30 \mathrm{Da}$ e $60 \mathrm{Da}$ a mais. Estes dados permitem atribuir aos compostos 2 e $\mathbf{3}$ a presença adicional de uma e duas metoxilas, respectivamente. Os íons produtos característicos para $2 \mathrm{em} \mathrm{m} / \mathrm{z}, 289,1184$ [M-H-(4-etenilfenol)]', $m / z, 259,1081$ [M-H-(5-etenil-metoxi-fenol)]', $m / z, 274,0939$ [M-H- $\mathrm{CH}_{3}$-(4-etenil-fenol)] e $\mathrm{m} / \mathrm{z}$ 162,8399 [M-H-feruloil- $\mathrm{C}_{4} \mathrm{H}_{7} \mathrm{~N}$ ] permite identificar 2 como sendo o conjugado coumaroil-feruloil-putrescina. O composto 3 foi identificado como di-feruloil-putrescina e apresentou os íons produtos $\mathrm{m} / z, 289,1184$ [M-H-(5-etenil-metoxifenol)] e $m / z$ 149,0597 [M-H-feruloil-putrescina-CO-CH$]^{-}$.

Os picos 4 e 6 apresentaram os picos em $\mathrm{m} / z$ 614,2501 [M-H] $]^{-} \mathrm{e}$ $\mathrm{m} / \mathrm{z}, 582,2607$ [M-H] $^{-}$, respectivamente. Os padrões de fragmentação destes compostos (Tabela 3 ) são característicos de espermidina ligada a derivados de ácidos cinâmicos. O composto 4 apresentou o íon produto em $\mathrm{m} / \mathrm{z}, 358,1376$ [M-H] $]^{-}$, que indica a presença de dois grupos cafeoil (2x179 Da), e o composto 6 mostrou o íon produto em $\mathrm{m} / z$ 162,8391 [M-H] $]^{-}$, característico de um grupo coumaroil. Com estes dados a substância 4 foi identificada como coumaroil-di-cafeoil espermidina e o $\mathbf{6}$ como $N^{1}, N^{5}, N^{10}$-tricoumaroil espermidina.

Os fenólicos 5, 7, 8 e 9 foram tentativamente identificados como biflavonóides. O pico $8 \mathrm{em} \mathrm{m} / z 537,0827$ [M-H] ${ }^{-}$produziu os íons produtos em $m / z$ 443,0394 [M-H-C $\left.\mathrm{C}_{7} \mathrm{H}_{6} \mathrm{O}_{3}\right]^{-}, m / z, 417,0592\left[\mathrm{M}-\mathrm{H}-\mathrm{C}_{7} \mathrm{H}_{6} \mathrm{O}_{3}\right]-$, $m / z, 375,0483$ [M-H- $\mathrm{C}_{10} \mathrm{H}_{6} \mathrm{O}_{5}$ ], $m / z, 309,0384$. O íon $m / z, 443,0394$ é o resultado da perda neutra do fenol da parte II do flavonoide (Espectro e fragmentos ionicos demonstrado na Figura 9S-A e B). O íon produto em $m / z, 375,0483$ [M-H- $\mathrm{C}_{10} \mathrm{H}_{6} \mathrm{O}_{5}$ ] é devido a retrociclização envolvendo a parte II do flavonoide (Figura 9S-B). Os dados de massas estão de acordo com Yao et al.$^{38}$ e permitem identicar o biflavonóide $\mathbf{8}$ como sendo a amentoflavona. Os biflavonóides 5 e 7 são derivados da amentoflavona, sendo que o $5 \mathrm{~m} / z$ 555,0934 [M-H]' apresentou uma hidroxila adicional e o $7 \mathrm{~m} / z, 539,0994$ [M-H] - dois hidrogenios a mais que amentoflavona (8). Os espectros de massas estão nas Figuras 8S (A e B) e 10S (A e $\mathrm{B})$, respectivamente. $\mathrm{O}$ biflavonóide 7 é o composto mais abundante na fração acetato de etila do pólen apícola de Fabaceae e foi tentativamente identificado como sendo 2,3-dihidro-amentoflavona. A posição da ligação dihidro entre os carbonos 2 e 3 dos dois biflavonoides ( 5 e 7 ) podem estar localizadas tanto na unidade do flavonoide I ( 2 e 3 ) quanto em II (2" e 3") que constituem os biflavonoides, não sendo possível definir a posição certa somente com os dados obtidos por massas. Os biflavonóides 5"'-hidroxiamentoflavona, 2,3-dihidroamentoflavona e amentoflavona já foram isolados e identificados nos grãos de pólen de Cryptomeria japonica. ${ }^{39}$

Tabela 1. Análises físico-químicas das amostras de polens apícolas (Média \pm D.P)

\begin{tabular}{|c|c|c|c|c|c|}
\hline \multirow{2}{*}{ Amostras } & Umidade & Cinzas & Atividade de Água & $\mathrm{pH}$ & Índice de acidez \\
\hline & Máx 4\%a & Máx 4\%a & - & $4 \mathrm{a}^{\mathrm{a}}$ & Máx $300 \mathrm{mEq} / \mathrm{kg}^{\mathrm{a}}$ \\
\hline Amostra I & $6,5 \pm 0,04$ & $1,5 \pm 0,44$ & $0,3 \pm 0,00$ & $5,2 \pm 0,02$ & $146,6 \pm 0,64$ \\
\hline Amostra II & $6,5 \pm 0,35$ & $2,0 \pm 0,35$ & $0,3 \pm 0,00$ & $5,1 \pm 0,01$ & $143,1 \pm 0,64$ \\
\hline
\end{tabular}

Tabela 2. Análise de açúcares no pólen apícola de Fabaceae

\begin{tabular}{|c|c|c|c|c|c|c|}
\hline \multirow{2}{*}{ Amostras } & \multicolumn{2}{|c|}{ Glicose } & \multicolumn{2}{|c|}{ Frutose } & \multirow{2}{*}{$\begin{array}{c}\mathrm{G}+\mathrm{F} \\
\mathrm{mg} \mathrm{g}^{-1}\end{array}$} & \multirow{2}{*}{$\mathrm{F} / \mathrm{G}$} \\
\hline & $\mathrm{mg} \mathrm{g}^{-1}$ & $\%$ & $\mathrm{mg} \mathrm{g}^{-1}$ & $\%$ & & \\
\hline Amostra I & $76,9 \pm 19,56$ & $7,7 \pm 1,96$ & $76,9 \pm 19,79$ & $7,7 \pm 1,98$ & 153,8 & 1,0 \\
\hline Amostra II & $104,6 \pm 6,40$ & $10,5 \pm 0,64$ & $103,4 \pm 1,97$ & $10,3 \pm 0,20$ & 208,0 & 1,0 \\
\hline
\end{tabular}


O biflavonóide $9 \mathrm{~m} / z, 539,0980$ [M-H] $]^{-}$é um isômero de 7. Os fragmentos ionicos permitem tentativamente identificar $\mathbf{9}$ como sendo 2,3-dihidro-biapigenina. Possivelmente a ligação pode ser do tipo IC3'-IIC3"'. Esta afirmação é suportada pelos fragmentos ionicos obtidos quando comparados com o mesmo composto reportado por Yao et al. ${ }^{38}$ Os íons produtos em $\mathrm{m} / \mathrm{z} 413,0647$ e $\mathrm{m} / \mathrm{z}$ 387,0857 são devido a ruptura da ligação por Retro-Diels-Alder (RDA) comum em flavonoides (Figura 10S-B). Estes dados estão de acordo com Yao et al..$^{38}$ De forma semelhante que os compostos 5 e $\mathbf{7}$, a posição da ligação dihidro (2,3 ou 2",3") não pode ser definida com os dados somente de massas para o composto 9.

Os compostos do tipo acil (derivados do ácido cinâmico) conjugados com esperminas e espermidinas já foram relatados em pólen apícola. Os derivados $N^{1}, N^{5}, N^{10}$-tri- $p$-coumaroil espermidina, ${ }^{40}$ $N^{1}, N^{5}, N^{10}, N^{14}$-tetra- $p$-coumaroil espermina e monocafeoil-tri- $p$-coumaroil espermina foram isolados de pólen apícola brasileira. ${ }^{41} \mathrm{Já}$ os derivados de acil-espermidinas: $N$ ', $N$ ", $N$ ' ',-tris-cafeoil espermidina, $N$, $N$ "-dicafeoil, $N$,"-coumaroil espermidina $N$, $N$ "'-dicafeoil, $N$,'”feruloil espermidina, $N$ '-cafeoil- $N$ "'-feruloil, $N$ '”-coumaroil espermidina $N$ '-cafeoil- $N$ ", $N$ ' "'- dicoumaroil espermidina, $N$,,$N$ ", $N$ ',-tris$p$-coumaroil espermidina, $N^{\prime}, N$ ", $N$,"'-tris-p-feruloil espermidina ${ }^{42}$ foram identificados em uma amostra de pólen apícola de coqueiro coletado na cidade de Canavieiras, Bahia.

Espécies da família Fabaceae são conhecidas por apresentarem, além de flavonoides, biflavonoides e bichalconas. Em relação à presença de biflavonoides em Fabaceae de ocorrência na região semiárida do Nordeste brasileiro, recentemente foram isolados novos biflavonóides das raízes do caule de Poincianella pyramidalis, uma espécie endêmica da caatinga ${ }^{43}$ e das folhas de Caesalpinia pyramidalis. $^{44}$

Os métodos utilizados para a medida da atividade antiradicalar foram com os radicais livres ABTS e DPPH, considerados sensíveis e precisos-pois o sistema de reação envolve apenas o radical e o antioxidante e são bastante utilizados para determinar a atividade antioxidante em alimentos e amostras biológicas. ${ }^{45} \mathrm{Na}$ Tabela 4 os resultados obtidos demonstram que a amostra II apresentou maior potencial antioxidante, apresentando maior teor de fenólicos e menor $\mathrm{CE}_{50}$ no ensaio DPPH e ABTS.

O pólen de abelha e outros produtos como mel e geoprópolis tem tido o seu potencial antioxidante atribuído a quantidade de conteúdo fenólico, principalmente flavonoides, cuja variação é relacionada com a origem das espécies botânicas que caracterizam estes produtos. ${ }^{24,46-50}$ Meneses et al..$^{51}$ estudaram a neutralização do radical DPPH de amostras pólen apicola do tipo Mimosa pudica apresentando significativa atividade sequestrante de radicais. Moreira et al. ${ }^{52}$ demonstraram que a concentração de fenólicos totais na própolis portuguesa apresentou uma forte correlação com a atividade antioxidante. Fatrcová-Šramková et al. ${ }^{53}$ comparando pólen apícola de papoula com pólen de abelha de girassol, relataram que o de papoula, com maior teor de polifenóis, apresentou maior atividade antibacteriana. Carpes et al. ${ }^{49}$ analisando amostras de pólen coletadas nas cidades de Contagem (MG), Saúde (BA),

Tabela 3. Caracterização dos compostos do pólen apícola de Fabaceae por UHPLC-DAD-QTOF-MS-MS

\begin{tabular}{|c|c|c|c|c|c|c|c|}
\hline & $\mathrm{TR}$ & $\lambda_{\max }$ & {$[\mathrm{M}-\mathrm{H}]^{-}$} & {$[\mathrm{M}-\mathrm{H}]^{-}$calculado } & Erro (ppm) & $\mathrm{MS} / \mathrm{MS}$ & Identificação \\
\hline 1 & 3,90 & 293 & 379,1661 & 379,1663 & 0,5 & $\begin{array}{c}\text { 259,1336 [M-H-(4-etenil- } \\
\text { fenol)] }]^{-}, 233,1336[\mathrm{M}-\mathrm{H}-\mathrm{cou}- \\
\text { maroil] }{ }^{-}, 162,0560[\mathrm{M}-\mathrm{H}- \\
\left.\text { coumaroil- }{ }_{4}{ }_{4} \mathrm{H}_{7} \mathrm{~N}\right]-\end{array}$ & Di-4-coumaroil-putrescina \\
\hline 2 & 4,10 & 308 & 409,1764 & 409,1768 & 0,9 & $\begin{array}{l}\text { 289,1184 [M-H-(4-etenil- } \\
\text { fenol)]- } 274,0939 \text { [M- } \\
\mathrm{H}-\mathrm{CH}_{3} \text {-(4-etenil-fenol)]; } \\
\text { 259,1081 [M-H-(4-etenil- } \\
\text { metoxi-fenol)]-; 162,8399 } \\
\left.\text { [M-H-feruloil- }{ }_{4} \mathrm{H}_{7} \mathrm{~N}\right]-\end{array}$ & Coumaroil-feruloil-putrescina \\
\hline 3 & 4,27 & 317 & 439,1870 & 439,1874 & 0,9 & $\begin{array}{l}289,1184 \text { [M-H-(4-etenil-me- } \\
\text { toxi-fenol)]; } 149,0597[\mathrm{M}-\mathrm{H}- \\
\text { feruloil-putrescina-CO-CH}]^{-}\end{array}$ & Di-feruloil-putrescina \\
\hline 4 & 4,30 & 314 & 614,2501 & 614,2507 & 0,9 & $\begin{array}{c}\text { 478,1979 [M-H-(4-etenil- } \\
\text { hidroxi-fenol)]; 452,2225 } \\
\text { [M-H-cafeoil]; } 289,1162 \\
\text { [M-H-dicafeil]- }\end{array}$ & Coumaroil-dicafeoil-espermidina \\
\hline 5 & 4,93 & 296,326 & 555.0934 & 555.0932 & $-0,3$ & $\begin{array}{c}527,1017[\mathrm{M}-\mathrm{H}-\mathrm{CO}]^{-}, 469,0927 \\
{\left[\mathrm{M}-\mathrm{H}-\mathrm{CO}_{2}-\mathrm{C}_{2} \mathrm{H}_{2} \mathrm{O}\right]^{-}, 351,0493} \\
{\left[\mathrm{M}-\mathrm{H}-\mathrm{CO}_{2}-3 \mathrm{C}_{2} 4 \mathrm{H}_{2} \mathrm{O}\right]^{-}} \\
309,0396\end{array}$ & 2,3-dihidro-hidroxi-amentoflavona \\
\hline 6 & 5,02 & 294 & 582,2607 & 582,2609 & 0,5 & $\begin{array}{c}\text { 462,2025 [M-H-(4-etenil- } \\
\text { fenol)]', } 342,1445[\mathrm{M}-\mathrm{H}-2 \mathrm{x}(4- \\
\text { etenil-feno })]-\end{array}$ & Tricoumaroil-espermidina \\
\hline 7 & 5,92 & 293 & 539,0994 & 539,0983 & $-2,0$ & $\begin{array}{c}\text { 495,1044 [M-H-CO } \mathrm{CO}_{2}^{-}, \\
453,0262\left[\mathrm{M}-\mathrm{H}-\mathrm{CO}_{2}-\mathrm{C}_{2} \mathrm{H}_{2} \mathrm{O}\right]^{-}, \\
351,0491\left[\mathrm{M}-\mathrm{H}_{-}-\mathrm{CO}_{2}^{-}\right. \\
\left.3 \mathrm{C}_{2} 4 \mathrm{H}_{2} \mathrm{O}\right]^{-}\end{array}$ & 2,3-dihidro-amentoflavone \\
\hline 8 & 6,08 & 267,336 & 537,0827 & 537,0827 & 0,0 & $\begin{array}{c}443,0394\left[\mathrm{M}-\mathrm{H}-\mathrm{C}_{7} \mathrm{H}_{6} \mathrm{O}_{3}\right]^{-}, \\
417,0592\left[\mathrm{M}-\mathrm{H}-\mathrm{C}_{7} \mathrm{H}_{6} \mathrm{O}_{3}\right]- \\
375,0483\left[\mathrm{M}-\mathrm{H}-\mathrm{C}_{10} \mathrm{H}_{6} \mathrm{O}_{5}\right] \\
309,0384\end{array}$ & Amentoflavona \\
\hline 9 & 6,35 & 284,328 & 539,0980 & 539,0983 & 0,5 & $\begin{array}{l}413,0647 \text { (fragmento RDA); } \\
387,0857 \text { (fragmento RDA) }\end{array}$ & 2,3-dihidro-biapigenina \\
\hline
\end{tabular}


São Cristóvão (SE) e Maceió (AL), relacionaram que compostos bioativos além dos flavonoides são responsáveis pela atividade antioxidante.

Tabela 4. Teor de fenólicos totais e atividade sequestradora de radicais livres

\begin{tabular}{lccc}
\hline & $\begin{array}{c}\text { Fenólicos totais } \\
\left(\begin{array}{c}\mathrm{mg} \mathrm{GAE} \mathrm{g}^{-1} \\
\text { extrato })\end{array}\right.\end{array}$ & $\begin{array}{c}\mathrm{DPPH} \mathrm{CE}_{50} \\
\left(\mu \mathrm{g} \mathrm{mL}^{-1}\right)\end{array}$ & $\begin{array}{c}\mathrm{ABTS} \mathrm{CE}_{50} \\
\left(\mu \mathrm{gL}^{-1}\right)\end{array}$ \\
\hline Amostra I & $625,67 \pm 11,20$ & $979,3 \pm 3,90$ & $65,95 \pm 1,20$ \\
\hline Amostra II & $858,00 \pm 16,17$ & $120,80 \pm 1,23$ & $36,63 \pm 1,27$ \\
\hline Ácido ascórbico & & $45,6 \pm 1,81$ & \\
\hline Trolox & & $0,735 \pm 0,151$ \\
\hline
\end{tabular}

As amostras dos polens apícolas de Fabaceae apresentam em sua composição química a presença de flavonóides e conjugados acil-aminas. Os biflavonoides como a amentoflavona têm exibido uma ampla variedade de atividades biológicas, principalmente características sequestrantes de radicais livres, antiinflamatória e antimicrobiana..$^{50,54-56} \mathrm{Li}$ et al. ${ }^{57}$ sugerem que a amentoflavona pode efetivamente proteger contra o dano oxidativo ao DNA (ácido desoxirribonucleico), tendo seu efeito protetor atribuído principalmente a sua capacidade antioxidante devido a porção fenólica. Para as poliaminas presentes têm-se atribuído propriedades antioxidantes, agindo sobre espécies envolvidas na peroxidação lipídica de ácidos graxos poliinsaturados..$^{58,59}$ Drolet et al. ${ }^{60}$ também observaram que espermina, espermidina, putrescina e cadaverina são eficientes sequestradores de radicais livres gerados em vários sistemas químicos e enzimáticos in vitro. Os compostos derivados de ácidos cinâmicos conjugados com espermidinas isolados do pólen apícola brasileiro já são conhecidos por apresentar atividades antiradicalar e antioxidante. ${ }^{40}$ Os compostos monocafeoil-espermina mostrou alta atividade antiradicalar e tri- $p$-coumaroil-espermidina apresentou forte atividade antioxidante. ${ }^{41}$

O estudo da atividade antimicrobiana de pólen apícola tem despertado interesse. Amostras de pólen de Castanea sativa coletadas na região do Mar Negro, Turquia, apresentaram atividade antimicrobiana seletiva e eficaz contra as cepas bacterianas e de leveduras avaliadas. ${ }^{61}$ Basim et al. ${ }^{62}$ avaliaram a atividade antibacteriana do pólen da Turquia contra diferentes bactérias patogênicas que causam doenças a frutas e vegetais, propondo o uso do pólen como agente protetor no manejo de culturas na agricultura. ${ }^{62}$ Morais et al.,${ }^{19}$ analisando pólen apícola heterofloral na região de Portugal, concluiram que não houve relação entre conteúdos fenólicos e atividade antimicrobiana.

A Tabela 5 mostra os resultados que demonstram que a fração AcOEt do pólen apícola de Fabaceae apresenta atividade frente à cepas de bactérias e fungos. A amostra II, com maior conteúdo fenólico e maior atividade sequestrante de radicais, também apresentou os melhores resultados frente às bactérias. Nossos resultados corroboram aos encontrados por Carpes et al., ${ }^{63}$ nos quais extratos de polens em diferentes concentrações etanólicas exibiram diferentes atividades antioxidantes e antibacterianas, o que foi relacionado com o diferente conteúdo fenólico extraído. Alguns estudos também têm sugerido a relação da atividade antimicrobiana com o total de compostos fenólicos presentes. ${ }^{47,52} \mathrm{~A}$ atividade antimicrobiana encontrada pode ser justificada pela presença de poliaminas conjugadas com derivados de ácidos cinâmicos e biflavonoides. Nayaka et al., ${ }^{64}$ avaliando a atividade antimicrobiana da apigenina, constataram que o flavonoide foi mais ativo contra bactérias Gram-positivas do que Gram-negativas, atribuindo essa diferença a diversas razões tais como complexidade da parede celular ou a presença de enzimas no espaço periplasmático. Morimoto et al. $^{65}$ indicam a apigenina como um novo antibiótico capaz de ser alternativa a resistência a quinolonas. As poliaminas biológicas, como a espermidina e putrescina, têm tido comprovada eficácia contra vários microorganismos, ${ }^{66,67}$ embora estudos sugiram que também estejam envolvidos em algumas rotas de defesa dos próprios microorganismos contra antibióticos. ${ }^{68,69}$

\section{CONCLUSÃO}

As análises palinológicas mostraram que a origem botânica predominante nas duas amostras de polens são da família Fabaceae. Pela análise de UPLC-DAD-qTOF-MS/MS foi possível identificar nove principais substâncias, sendo três derivados da putrescina (di-4-coumaroil-putrescina, coumaroil-feruloil-putrescina e di-feruloil-putrescina), dois derivados da espermidina (coumaroil-dicafeoil-espermidina e tricoumaroil-espermidina e quatro biflavonoides (2,3-dihidro-hidroxi-amentoflavona, 2,3-dihidro-amentoflavone, amentoflavona, 2,3-dihidro-biapigenina). As duas amostras apresentaram atividades antiradicalares e antimicrobianas, provavelmente devido à presença destes compostos. O pólen apícola já considerado alimento funcional apresenta potencial para a produção de produtos tecnológicos com valor nutricional, rico em antioxidantes e com capacidade antimicrobiana, uma matriz importante para a indústria alimentícia e farmacêutica.

\section{MATERIAL SUPLEMENTAR}

Cromatogramas e espectros de massas MS/MS das análises (Figuras 1S-A e B a 10S-A e B, ) estão disponíveis em http://www. quimicanova.sbq. org.br, em formato PDF, com acesso livre.

\section{AGRADECIMENTOS}

Este projeto foi financiado pelo CNPq-Renorbio-Pindorama (Processo número 407659/2013-7) e FACEPE-PRONEM (Processo número 0741.1.06/14). Os autores agradecem ainda à CAPES e ao CENAPESQ-UFRPE.

Tabela 5. Atividade antimicrobiana das frações acetato de etila do pólen apícola de Fabaceae

\begin{tabular}{lcccc}
\hline \multirow{2}{*}{ Microorganismo } & \multicolumn{3}{c}{ Concentração Inibitória Mínima $(\mathrm{CIM}, \mu \mathrm{g} / \mathrm{mL})$} \\
\cline { 2 - 5 } C. albicans ATCC 36232 & Fabaceae I & Fabaceae II & Cetoconazol & Meropenem \\
C krusei ATCC 34135 & 512 & 512 & $\leq 16$ & - \\
C tropicalis ATCC 13803 & $>1024$ & 1024 & $\leq 16$ & - \\
C glabrata ATCC 90030 & 1024 & $>1024$ & $\leq 16$ & - \\
S. aureus ATCC 6538 & 256 & 512 & - & 4 \\
E. coli ATCC 25922 & 128 & 64 & - & 4 \\
\hline
\end{tabular}




\section{REFERÊNCIAS}

1. Bogdanov, S.; Pollen: Production, Nutrition and Health - A Review; Bee Product Science, www.bee-hexagon.net, 2017.

2. Instrução Normativa $\mathrm{N}^{\circ} 3$, Ministério da Agricultura, Pecuária e do Abastecimento, 19 de janeiro de 2001, publicado no Diário Oficial da União em 23 de janeiro de 2001.

3. Barreto, L. M. C.; Funari, S. R. C.; Orsi, R. O.; Bol. Ind. Anim. 2005, 62, 167.

4. Paula-Neto, F. L.; Almeida-Neto, R. M. A.; Apicultura nordestina: principais mercados, riscos e oportunidades, Série Documentos do ETENE n. 12, Banco do Nordeste do Brasil: Fortaleza, 2006.

5. Novais, J. S.; Lima, L. C. L.; Santos, F. A. R.; Grana 2009, 48, 224.

6. Freire, K. R. L.: Lins, A. C.; Dórea, M. C.; Santos, F. A.; Camara, C. A.; Silva, T. M. S.; Molecules 2012, 17, 1652.

7. Morgano, M. A.; Martins, M. C. T.; Rabonato, L. C.; Milani, R. F.; Yotsuyanagi, K.; Rodriguez-Amaya, D. B.; J. Braz. Chem. Soc. 2012 23, 727.

8. Yang, L.; Yan, Q. H.; Ma, J. Y.; Wang, Q.; Zhang, J. W.; Xi, G. X.; Trop. J. Pharm. Res. 2013, 12, 771 .

9. Silva, G. R.; Tese de Doutorado, Universidade Federal Rural de Pernambuco, Brasil, 2014.

10. Feás, X.; Vázquez-Tato, M. P.; Estevinho, L.; Seijas, J. A.; Iglesias, A.; Molecules 2012, 17, 8359.

11. Komosinska-Vassev, K.; Olczyk, P.; Kazmierczak, J.; Mencner, L.; Olczyk, K.; Evid. Based Complement. Alternat. Med. 2015, ID 297425.

12. Arruda, V. A. S. A.; Pereira, A. A. S.; Freitas, A. S.; Barth, O. M. B.; Almeida-Muradian, L. B.; J. Food Comp. Anal. 2013, 29, 100.

13. Campos, M. G. R.; Bogdanov, S.; Almeida-Murdian, L. B.; Szczesna, T.; Manceo, Y.; Frigerio, C.; Ferreira, F.; J. Apic. Res. 2008, 47, 154.

14. Carpes, S. T.; Alencar, S. M.; Begnini, R.; De Alencar, M. S.; Masson, M. L.; Ciênc. Agrotec. 2007, 31, 1818.

15. Carpes, S. T.; Alencar, S. M.; Cabral, I. S. R.; Oldoni, T. L. C.; Mourão, G. B.; Haminiuk, C. W. I.; Luz, C. F. P.; Masson, M. L.; CyTA--J. Food 2013, 11, 150 .

16. Ulusoy, E.; Kolayli, S.; J. Food Biochem. 2013, 38. 73.

17. Mohdaly, A. A. A.; Mahmoud, A. A.; Roby, M. H. H.; Smetanska, I.; Ramadan, M. F.; J. Food Biochem. 2015, 39, 538.

18. Pascoal, A.; Rodrigues, S.; Teixeira, A.; Feás, X.; Estevinho, L. M.; Food Chem. Toxicol. 2014, 63, 233.

19. Morais, M.; Moreira, L.; Estevinho, L. M.; Food Chem. Toxicol. 2011, 49, 1096.

20. Silva, T. M. S.; Camara, C. A; Da Silva, L. A.; Barbosa-Filho, J. M.; Da Silva, E. M. S.; Freitas, B.; Santos, F. A. R.; J. Food Compos. Anal. 2006, $19,507$.

21. Silva, T. M. S; Camara, C. A.; Lins, A. C.; Agra, M. F.; Silva, E. M. S.; Reis, I. T.; Freitas, B. M.; An. Acad. Bras. Ciênc. 2009, 81, 173.

22. Silva, T. M. S.; Santos, F. P.; Rodrigues, A.; Silva, E. M. S.; Silva, G. S.; Novais, J. S.; Santos, F. A. R.; Camara, C. A.; J. Food Compos. Anal. 2013, 29, 10.

23. Da Silva, I. A. A.; Silva, T. M. S.; Camara, C. A.; Queiroz, N. M. M.; De Novais, J. S.; Soledade, L. E. B.; Lima, E. O.; De Souza, A. L.; De Souza, A. G.; Food Chem. 2013, 141, 3552.

24. Souza, S. A.; Camara, C. A.; Silva, E. M. S.; Silva, T. M. S.; Evid. Based Complement. Alternat. Med. 2013, ID 801383.

25. Alves de Souza, S.; Silva, T. M. G.; Camara, C. A.; Silva, E. M. S.; Silva, T. M. S.; Phytochem. Anal. 2018, 29, 1.

26. Silva, T. M. S.; Souza, S. A.; Dias, T. L. M.; Camara, C. A.; Sociobiology 2014, 61, 560.

27. Silva, G. R.; Natividade, T. M.; Camara, C. A.; Da Silva, E. M. S.; Santos, F. A. R.; Silva, T. M. S.; Food Nutr. Sci. 2014, 5, 1015.

28. Erdtman, G.; Sven. Bot. Tidskr. 1960, 54, 561.

29. Singleton, V. L.; Joseph, A.; Rossi, J.; Am. J. Enol. Vitic. 1965, 16, 144.
30. Metodologia dos Testes de Sensibilidade a Agentes Antimicrobianos por Diluição para Bactéria de Crescimento Aeróbico, National Committee for Clinical Laboratory Standards, 2005, p. 23.

31. Menezes, J. D. S.; Maciel, L. F.; Miranda, M. S.; Druzian, J. I.; Rev. Inst. Adolfo Lutz. 2010, 69, 233.

32. Fabaceae in Flora do Brasil 2020, under construction, Jardim Botânico do Rio de Janeiro. Disponível em http://floradobrasil.jbrj.gov.br/reflora/ floradobrasil/FB115, acessado em Setembro 2018.

33. Matos, V. R.; Alencar, S. M.; Santos, F. A. R.; An. Acad. Bras. Cienc. 2014, 86, 407.

34. Anderson, G. J.; Bernardello, G.; Stuessy, T. F.; Crawford, D. J.; Am. J. Bot. 2001, 88, 220.

35. Cane, J. H.; Gardner, D. R.; Harrison, P. A.; Apidologie. 2011, 42, 401.

36. Bertoncelj, J.; Polak, T.; Pucihar, T.; Lilek, N.; Kandolf, B.; Korosec, M.; Int. J. Food Sci. Technol. 53, 1880.

37. Elejalde-Palmett, C.; Bernonville, T. D.; Glevarec, G.; Papon, O. P. N.; Courdavault, V.; St-Pierre, B.; Lanoue, N. G. A.; Besseau. S.; J. Exp. Bot. 2015, 66, 7271 .

38. Yao, H.; Chen, B.; Zhang, Y.; Ou, H.; Li, Y.; Li, S.; Shi, P.; Lin, X.; Molecules 2017, 22, 325.

39. Ohmoto, T.; Yoshida, O.; Chem. Pharm. Bull. 1983, 31, 919.

40. Negri, G.; Teixeira, E. W.; Alves, M. L. T. M. F.; Moreti, A. A. C. C.; Otsuk, P. I.; Borguini, R. G.; Salatino, A.; J. Agric. Food Chem. 2011, $59,5516$.

41. Ohta, H.; Fujimaktz, T.; Uyz, M. M.; Yanaix, M.; Yukiyoshi, A.; Hirata, T.; Nat. Prod. Res. 2007, 21, 726.

42. Negri, G.; Barreto, L. V. R. C.; Sper, F. L.; Carvalho, C.; Campos, M. G. R.; Braz. J. Food Technol. 2018, e2016176.

43. Oliveira, J. C.; David, J. P.; David, J. M.; Phytochem. Lett. 2016, 18.

44. Bahia, M. V.; Santos, J. B.; David, J. P.; David, J. M.; J. Braz. Chem. Soc. 2005, 16, 1402.

45. Boylan, F.; Menezes, S.; Leita, G. G.; Phytother. Res. 2015, 130, 27.

46. Souza, S. A.; Silva, T. M. G.; Camara, C. A.; Silva, E. M. S.; Silva, T. M. S.; Phytochem. Anal. 2018, $29,1$.

47. Araújo, J. S.; Chambó, E. D.; Costa, M. A. P. C.; Cavalcante, S, S. M. P.; Lopes, C. C. A.; Estevinho, L.; Int. J. Mol. Sci. 2017, 18, 921.

48. Da Cunha, M. G.; Franchin, M.; Galvão, L. C.; De Ruiz, A. L.; De Carvalho, J. E.; BMC Complement. Altern. Med. 2013, 13, 242.

49. Carpes, S. T.; De Alencar, M. S.; Masson, M. L.; Braz. J. Food Technol. 2009, 12, 220.

50. Ferreira, R. O.; De Carvalho, M. G.; Silva, T. M. S.; Quim. Nova 2012, $35,2271$.

51. Meneses, J. D. D. S.; Maciel, L. F.; Miranda, M. S.; Druzian, J. L.; Rev. Inst. Adolfo Lutz 2010, 69, 233.

52. Moreira, L.; Dias, L. G.; Pereira, J. A.; Estevinho, L.; Food Chem. Toxicol. 2008, 46, 3482.

53. Fatrcová-Šramková, K.; Nôžková, J.; Kačániová, M.; Máriássyová, M.; Rovná, K.; Stričík, M.; J. Environ Sci. Health, Part B 2013, 48, 133.

54. González-Gallego, J.; Sánchez-Campos, S.; Tunon, M.; J. Nutr. Hosp. 2007, 22, 287.

55. Kim, H. P.; Son, K. H.; Chang, h.W.; Kang, S. S.; J. Pharmacol. Sci. 2004, 96, 229.

56. Lima, R. R.; Costa, A. M. R.; Souza, R. D.; Gomes-Leal, W.; Rev. Para. Med. 2007, 21, 29.

57. Li, X. Li.; Wang, L.; Han, W.; Mai, W.; Chen, D.; Turk J. Biochem. 2014, 39, 30.

58. Lovaas, E.; Carlin, G.; Free Radical Biol. Med. 1991, 11, 455.

59. Stevanato, R.; Bertelle, M.; Fabris, S.; Pharmacol. Pharm. 2013, 4, 696.

60. Drolet, G.; Dumbrof, E. B.; Legge, R. L.; Thompson, J. E.; Phytochemistry 1986, 25, 367.

61. Avsar, C.; Ozler, H.; Berber, I. Civek, S.; Int. Food Res. J. 2016, 23 , 1711.

62. Basim, E.; Basim H.; Özcan M.; J. Food. Eng. 2006, 77, 992. 
63. Carpes, S. T.; Begnini, R.; Alencar, S. M.; Masson, M. L.; Ciênc. Agrotec. Lavras. 2008, 31, 1818.

64. Nayaka, H. B.; Londonkar, R. L.; Umesh, M. K.; Tukappa, A.; Int. J. Bacteriol. 2014, 2014, 175851.

65. Morimoto, Y.; Baba, T.; Sasaki, T.; Hiramatsu, K.; Int. J. Antimicrob. Agents. 2015, 46, 666.

66. Grossowicz, N.; Razin, S.; Rozansky, R.; J. Gen. Microbiol. 1955, 13, 436.
67. Rayavarapu, S.; Kadiri, S.; Basha, M.; Mangarao, N.; Tadikondal, L.; Sastry, N.; Vidavalur, S.; Med. Chem. 2014, 4, 367.

68. El-Halfawy, O. M.; Valvano, M. A.; Antimicrob. Agents Chemother. 2014, 58,4162 .

69. Johnson, L.; Mulcahy, H.; Kanevets, U.; Shi, Y.; Lewenza, S.; J. Bacteriol. 2012, 194, 813. 